\title{
Integrating a 3D Body Scanner into an Active Bariatric Surgery Clinic Practical Experiences, History, Tips and Pitfalls
}

\author{
David B. STEFAN ${ }^{1}$, Stephen D. WOHLGEMUTH ${ }^{2,3}$, David A. GILBERT ${ }^{4}$ \\ ${ }^{1}$ Novaptus Systems, Inc. Chesapeake, VA, USA; \\ ${ }^{2}$ Eastern Virginia Medical School, Norfolk, VA, USA; \\ ${ }^{3}$ Sentara Comprehensive Weight Loss Solutions, Norfolk, VA, USA; \\ ${ }^{4}$ The Hague Plastic and Cosmetic Surgery Center, Norfolk, VA, USA
}

http://dx.doi.org/10.15221/14.124

In late 2005, a 3D whole body scanner began to be used to measure morbidly obese (bariatric) patients prior to surgery. This scanner was located in a cosmetic surgeon's office. The volume of scans for these bariatric patients soon began to overwhelm the cosmetic surgery practice's staff. In early 2007, a 3D scanner was placed directly into the bariatric surgery clinic. Protocols were written to streamline and define the bariatric clinic's staff involvement and to seamlessly integrate the scanner into the clinic's daily operations. Meaningful reports were designed and a mechanism was created to distribute them to the patients. A billing system was also introduced. Since that time, improvements to scanner technology has led to several scanner replacements, with only slight changes to the overall protocol framework.

\section{Introduction}

To tell the reader that this is a scientific paper presenting the statistical results of applying a 3D whole body scanner to measure a sample of the morbidly obese population or subjects that underwent a cosmetic surgery procedure would be misleading. Those papers and their associated presentations do exist, and the reader is encouraged to look up the other authors of this paper to find them. Rather, this is a narrative about pioneering deployment of $3 \mathrm{D}$ scanning systems into two distinct medical environments, from scratch, and with no previous guidelines to act as signposts along the way. As such, you will not find a reference section at the end of this paper.

It is a history of an idea. It is a journey of trial and error... a lot of errors, actually. Some of these errors were self-inflicted, others simply appeared along the way. But this is how one learns. It has been said that "the pioneers take all the arrows, and the settlers take the land". In that vein, this paper offers insight and advice to those looking to deploy a 3D body scanner into a medical environment. Some of this advice may fall under the "well that's obvious" category. Some of the advice may also fall into the "it will never happen to me" bin. Don't be fooled. The big picture is important, but you will find, as we had to, that even the smallest stuff really does matter.

\section{Background}

\section{The early years}

The idea of using a 3D body scanner in a medical practice was conceived by a plastic surgeon traveling on an aircraft during holiday in 2001. The surgeon was reading a copy of the Wall Street Journal. An article in that paper discussed an experience the reporter encountered when using a 3D, whole body scanner for custom fitted clothes that was located in the Fashion District of New York City. The reporter and the article were not exactly complementary to the scanning process. She complained about the cleanliness of the scan chamber and her scan experience, but she did admit that the clothing produced by the scanner measurements fit her better than clothing that was available "off the rack".

To the plastic surgeon, that wasn't the point. Here was a device that might be able to better assess the body contours of his patients, and the results of the surgical procedure by way of consistent and repeatable measurements. Body contouring procedures, as the term suggests, are surgeries aimed to affect, with an eye to improve, a patient's physical appearance. Surgical procedures such as lipoplasty, abdominoplasty (aka "tummy tuck"), breast reduction and breast augmentation are popular examples. All these procedures result in some sort of physical change to the body, which could conceivably be measured and evaluated by capturing preoperative scans and postoperative scans of a patient and comparing the measurement differences. 
Surgical body contouring procedures, as a plastic surgeon would admit, are an equal part of medical training and surgical experience and are far from formulaic in nature. In 2001, it was common to still take $35 \mathrm{~mm}$ pictures and have them produced as slides. These formed the essence of the "before and after" library, as well as the image repository of a primitive medical archive to document surgical results. Of course, these types of photographs were not consistent and repeatable. Patients would stand in different positions and at different focal lengths and this made exact comparisons between the two analog photos difficult.

Ideas about how a 3D body scanner might be utilized in a medical practice kept churning within the plastic surgeon's mind. Further research led him to the offices of Textile Clothing Technology Corporation, or TC(2), which he visited in early 2002. At that time, the 3D body scanner consisted of four large mechanical projectors mounted on two metal frames, one on top of the other. The dimensions of this scanner model were 8 feet long by 16 feet wide. The entire scanning apparatus was enclosed in cloth, including the ceiling cover. These mechanical projectors traveled along a fixed distance on the $y$-axis. Light from the projectors would travel through various grid patterns as they moved up and down. This light would be reflected off the subject and would be captured as a series of photographs by the projector's cameras. This technique was generally known as "White Light Phase Profilometry"..

Picture the situation through the plastic surgeon's perspective. Here was new technology that might be able to improve pre-operative patient evaluation, post-operative surgical results, and produce a 3D model of the patient before and after surgery that could be archived in digital format. But this scanner had a large footprint. It wouldn't fit into a standard sized examination room. No one had any expertise in taking scans, let alone setting the scanner up or calibrating and maintaining the system. What was to be done with the information produced? How was it going to be evaluated? Who would operate the scanner? How much time would be taken out of the clinic's staff?

These early scanners had a very large price tag, too.

Throughout the spring of 2002 the plastic surgeon sought the means and the space to procure a scanner. Fortunately, the clinic had a large storage room that was underutilized. It was large enough to comfortably house the scanner. Shelves containing boxes of medical supplies would have to be moved, but otherwise it offered space and some privacy. In the summer of 2002, a chance visit occurred. A conversation took place on an elevator between the author and the plastic surgeon. The plastic surgeon spoke of his idea to locate the 3D scanner within his practice, and how it might be utilized. Intrigued, the author scheduled a visit to the scanner manufacturer's facilities and met with management in the latter part of the summer. It appeared that this was a unique opportunity to start something from scratch, since none of the scanners that were produced to that point had been deployed in a clinical practice.

Not too long after the visit to the scanner manufacturer, the plastic surgeon, his partner and the author decided to fund a start-up business that would, at the very least, place a scanner into the medical practice. Funds were pooled together, and a charter was created in September of 2002. The company purchased a scanner in late October, 2002. It was delivered and installed within the medical practice in November of 2002.

The first scan taken was, of course, of the plastic surgeon. Figure 1 displays a photo of the first scanner installed at a medical practice.

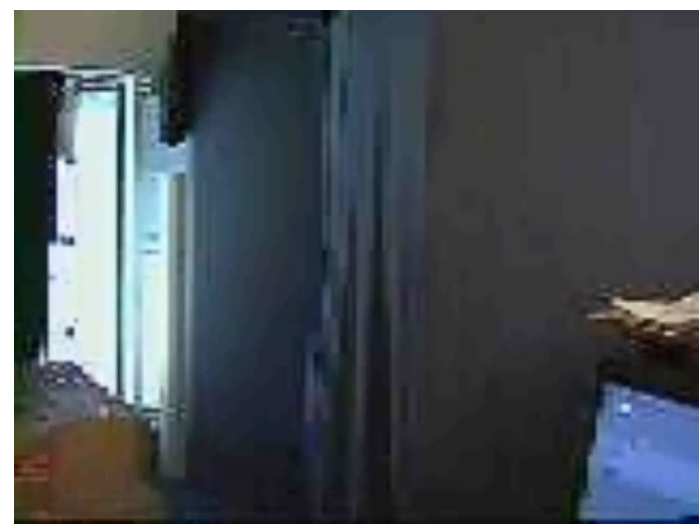

Figure 1. The Original 3D Scanner circa 2002 
Now that the idea of a scanner in the medical practice was realized, the next obvious question was: "What next?"

Was the scanner only to be used to assist the surgeon? What measurements would be needed? What measurements were available? How was the information to be formatted, processed and stored? Could the patient benefit by viewing any output? Could the 3D body model be used for predictive purposes? And how were the patients going to be scanned? How where the scans to be identified? How far must they disrobe? What about privacy in the scanner room while they prepare to be scanned? And then after they got scanned while they put their clothes back on? Who on the staff would take the scans? How were they to be trained? Who would be the support contact?

This was clearly a "green field" situation. And it was clearly going to be "trial and error" for some time. A framework was needed to guide the effort. But there wasn't a previous foundation to be built upon.

Several nurses were trained on how to take a scan, along with the plastic surgeon. The process was simple enough as long as a few guidelines were followed. We considered the scanner deployed within the plastic surgery practice more as an investigation into the scanner software's measurement capabilities, without regards to a specific product to be created or service to be performed. This was an informal, non-production environment. Such an environment allowed wide variability in process and procedures until a general, workable framework could be developed. But it also lacked certain focus. One point that became obvious was that there were so many varieties of body contouring procedures that procedure-specific measurement templates needed to be developed. A "one size fits all" measurement template would not be sufficient. This complicated preoperative and postoperative analysis of various body contouring procedures, but it forced us to think hard about naming conventions, data storage, retrieval and compartmentalization. It was apparent that there would be no quick answers as to how best to use the scanner's capabilities. Was the focus to be on breast-specific contouring procedures such as augmentation or reduction? Were physical contour changes from lipoplasty measurable? Could edema and its subsequent reduction be documented?

Efforts to utilize the scanner and all its measurement capabilities continued within the plastic surgery practice. All sorts of cases that presented were scanned pre-operatively and post-operatively, often on a repetitive basis. The most interesting cases appeared to be that of breast augmentation and breast reduction. These procedures resulted in immediate, large and noticeable changes to the physical contours of the patient. The scanner proved to be a fairly effective measuring tool for documenting significant changes to bust size. This was true whether the measurements extracted were circumferential, surface area or volumetric in nature. However, we also noticed that there would often be image dropouts on the sides of the breasts. This was a peculiar feature of the original scanner in that certain areas of the body lacked adequate camera coverage due to the camera configuration. In these cases, the scanner software would try to adjust for the image dropouts by forming a plane that connected through the dropout area with the nearest adjacent points within the body model. This was acceptable in most cases. However, the lack of coverage for certain sizes and shapes of breasts resulted in some gross measurement errors.

We began to realize that there were limitations on the scanner's ability to acquire images and measure certain areas of the body model. Some of the measurements extracted by the software differed from where the surgeon thought they should be. At that time, there was no ability to manually adjust the measurements points.

Collectively, we began to think beyond the confines of a plastic surgery practice and look out into the greater medical landscape. What other types of surgery would yield dramatic changes to the physical contours of a subject in a short period of time? Moreover, what type of surgery would yield changes to the entire subject's body? And could these changes be measured with consistency? 


\title{
The early years...lessons learned from the initial scanner installation
}

Don't overthink. Had we stopped to plan every detail about how the scanner was going to be used before purchase and installation, given the originality of the application, we would have had unrealistic expectations.

Have a flexible environment. The scanner was installed in a private practice. The clinical staff were cooperative and close-knit. Turnover was low. This allowed the ability to train the staff to take the scans, and to observe and adjust guidelines to quickly improve the acquisition process.

\begin{abstract}
Acknowledge that the scanner is just a machine, and all machines have limitations. Coverage of certain areas of the body were problematic, and some of the measurements automatically extracted were suspect. Most were satisfactory. An effort began to compile a list of measuring points that needed to be manually adjusted.

Inform the patient and have them sign consent forms. As part of the paperwork the patient filled out for their surgical procedure, they were shown a simple consent form that they were required to sign. The consent form included a brief description of the scanning process, and how the surgeon was going to use the information. The scan form explicitly mentioned that the scanner was a surface scanner that used harmless white light.

Discuss with the patient what to expect from the scanning experience. Interview the patient as to their scanning experience. Even though a medical practice has an air of authority and patients generally do as they are told by the doctor or the nurses, the feedback proved invaluable and it eventually led to many of the procedures that are now followed as part of the routine scanning process.
\end{abstract}

\section{Enter the bariatric surgeon....the mixed use years}

In 2005, by sheer coincidence, the author and the plastic surgeon were on a break in the surgeon's lounge of a local hospital. A bariatric surgeon was also present. We began discussing our use of the scanner within the plastic surgery arena and we asked a simple question: Would this device be of use to a bariatric surgery practice? The bariatric surgeon thought about this. Morbidly obese patients prior to bariatric surgery are rarely measured, with the exception of height and weight to calculate a Body Mass Index. It was decided that a simple placard would be placed on the receptionist's desk offering the patient the option to get a body scan and a measurement printout, but they would have to travel to the plastic surgery clinic to get this done.

The first preoperative bariatric patients were scanned in November of 2005. This was not a convenient process. The scanner was located at the plastic surgery clinic and the bariatric surgery practice was located several miles away. Preoperative bariatric patients, voluntarily, had to travel to the plastic surgery location, and the personnel at the plastic surgery center had to take the scans. They did this with no compensation. There was no fee charged at this time to either practice or a patient for the use of the scanner.

A straightforward measurement extraction profile was constructed. These consisted of common circumferential measurements that most people would want to know. Measurements such as hips, waist, bust or chest, biceps, thighs and neck were included in the measurement profile. We originally included both left and right wrists, but eliminated them after the first few scans due to coverage issues. This measurement extraction profile, or MEP, was applied to all the bariatric patients, regardless of gender. For the first time, a fixed MEP could be confidently utilized on a patient class. That MEP is still in use today. This is an important point. Scans processed nearly a decade ago do not need to be retrieved and measured again. Instead, by applying the same MEP file to all the bariatric patients, the measurements extracted are simply appended to a database file.

For the first 2 weeks of this program, only a few preoperative bariatric patients ventured to the plastic surgery center to get their scan. Of those that did, they received an immediate printout of their body measurements. The insight this revealed was astounding. We found out that preoperative bariatric patients really didn't know their physical size or even their physical shape. The most they might have known about their body measurements were perhaps their waist and hips, but even these measurements are not often taken prior to surgery. For the first time, these patients were "confronted" with their physical dimensions. We began to hear anecdotal comments from the patients such as "This is what happens when you let yourself go" (mostly from the males). Or, "I can't believe my 
husband still loves me", from many of the females. We also noticed that the scanner had difficulty extracting certain measurements from the bariatric patients' body models, particularly the arm measurements and sometimes abdominal measurements, including the "crotch point".

Over the following weeks and months, mostly by "viral contact" between preoperative bariatric patients at information seminars, more and more visited the plastic surgery center to get their body scan and their measurement printout. It got to the point where the staff at the plastic surgery center could no longer receive bariatric patients at random and continue to perform their own tasks.

It also got to the point where it was decided that the scans needed to be checked for accuracy prior to the bariatric patients receiving their printout. The bariatric patient was made to fill out a form that included their home address and their email address. Scans would be downloaded weekly on a memory stick at the plastic surgery center, and the forms would be picked up. The scans were processed in the data center, corrected for any errors if possible, then emailed or, if needed, mailed to the patient via the postal service.

This posed another issue: How best to separate and process scans from the same scanner for two distinct medical practices?

A coordinated effort between the two practices limited the time that bariatric patients could get scanned at the plastic surgery center to Wednesday afternoons. In spite of this constraint, the volume of bariatric patients coming to get scanned continued to increase. This started to become a problem for the plastic surgery clinic. And it was another lesson to be learned. Each Wednesday afternoon, a different employee at the plastic surgery clinic was designated to take the scans of the bariatric patients. At first, it was thought that the patients would come in at random between $1 \mathrm{pm}$ and $5 \mathrm{pm}$, and that the designated employee could still find time to perform their usual work.

As the volume of bariatric patients to be scanned continued to increase, the designated employee, no matter who it was, began to complain about "overload". It was a valid concern. The temporary solution was to have the bariatric patient call the plastic surgery clinic prior to arriving and schedule a scan appointment. Friction began to appear on the part of the plastic surgery clinic staff. At first it was muted, but then it became quite vocal. The effect of this was a noticeable drop in scan quality, both for the plastic surgery patients as well as the "intruding" bariatric patients. Even plastic surgery clinic personnel who were not associated with scanning began to complain about the increased "foot traffic". By the fall of 2006, preoperative bariatric patients began returning for their postoperative scans. This increased the volume of patients getting scanned and compounded the personnel problem.

Fortunately, two events occurred in late 2006 to relieve this situation, and they happened sequentially.

Recall that the original scanner that was purchased was quite large and required an expansive room. In the plastic surgery center, this room was a storage room in the back of the clinic. Patients, regardless of whether they were there for plastic surgery or bariatric surgery would have to walk through the clinic, into a busy corridor to enter the scan room. This was awkward and interfered with the normal flow of traffic within the plastic surgery clinic.

A new, improved scanner model, the NX-12 was introduced at that time. The NX-12 replaced the mechanical projector units with an array of fixed sensor heads placed at the corners of the scanner. Halogen light bulbs in the sensor heads replaced the previous light source. As important, the dimensions of the NX-12 scanner were reduced from 8 feet $\times 16$ feet to 5 feet $\times 12$ feet. There was an examination room adjacent to the reception desk that could, just barely, accommodate the new scanner footprint. The original scanner was replaced with the NX-12 scanner, which was relocated to this examination room. This improved traffic flow within the plastic surgery clinic, resulting in reduced complaints from certain staff members. The new scanner also captured and produced scan images at a slightly faster rate than the original mechanical scanner.

The new location for the scanner had complications, however, and did nothing to relieve the lobby congestion caused by the increasing volume of bariatric patients arriving to be scanned. Though the NX-12 scanner fit into the examination room, and could accommodate the majority of subjects, there was little room left over for furniture, especially the type of furniture sized for morbidly obese individuals.

Here was another lesson that was learned. "Convenience" furniture is necessary in the scan room. 


\section{The mixed use years...lessons learned from scanning two different sets of patients}

Do not underestimate the impact of the scanning process on clinic personnel. They have a full time job with other responsibilities to perform. Burdening them with additional responsibilities to the point of overload will yield poor results. This can carry over to other personnel and pose a threat to office morale.

Uncoordinated visits can led to waiting room congestion. No one likes disorganization. It can appear unprofessional. Either have a fixed schedule with dedicated personnel to accommodate patients that need to be scanned, or develop guidelines that seamlessly integrate scanning into the daily office routine.

Make the scanning environment as convenient as possible, for both the clinic personnel and the patient. Have adequate space with furniture such as a chair, clothes tree and a stand for the patients to place their belongings on when they undress. Have a mirror in the room so the patients can check their appearance after they put their clothes back on. A simple box of "dollar store" hair clips or bands is useful.

Privacy and communication are both essential. The scanner should be placed in a room with a door. The personnel taking the scan need to communicate with the patient to ensure that the patient follows the simple scanning rules and is in the scan chamber with the curtain drawn shut before entering the room. Set patient expectations about the scan process and give them adequate time to put their clothes back on.

A clear and simple to understand sign-in sheet is important. This helps identify the patient, gives the nurse instructions as to how to enter the name in the scan name field, and can be used for separating, processing and storing scan information in the data center.

Pay close attention to feedback from the nurses taking the scans and the patients themselves. Constantly try to improve and streamline the scanning process without sacrificing scan quality.

\section{The scanner integrated into the bariatric practice...the $\mathrm{nx}-16$ years}

The second event was unrelated to the NX-12 scanner. The bariatric surgery practice up to that point was an independent entity, not affiliated with any larger organization. In 2007, the practice was bought and merged into a large hospital system. The practice was to be moved to a large office section on a floor in a hospital. Here was a chance to design the room around a scanner to accommodate the morbidly obese.

In between the time that the bariatric practice was being subsumed into the hospital network and relocating to its new facility, the scanner manufacturer introduced a new scanner model: The NX-16. The NX-16 further reduced the scanner footprint from 5 feet $x 12$ feet to around 5 feet $x 6$ feet. It also increased the cameras from 12 to 16 , thereby improving coverage. This was fortuitous as the room that was designated in the hospital to house the scanner had dimensions that would just barely accommodate the older NX-12 scanner model. The NX-12 model scanner would remain at the plastic surgery center for a couple more years, eventually being upgraded to an NX-16. Figure 2 depicts the NX-16 scanner installed in a medical examination room. 


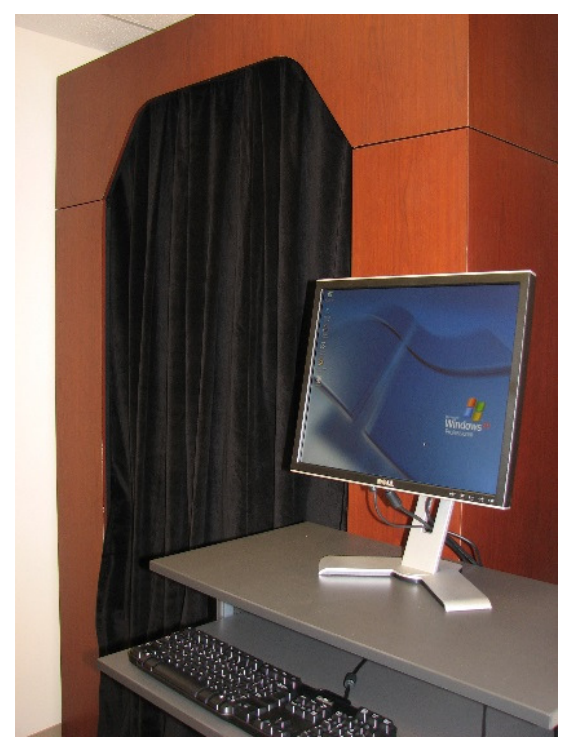

Figure 2. The NX-16 Scanning System Installed in a Medical Examination Room

Thus the bariatric practice that started scanning their patients by referring them to the plastic surgery clinic was going to receive their own scanner, to be operated by their own personnel. Here was an opportunity to establish guidelines that integrated the scanner into the everyday tasks performed by the bariatric clinic's staff.

The valuable experiences that the scanner at the plastic surgery center yielded over the early years would be incorporated into designing the guidelines for scanner usage at the bariatric practice.

First, you had to acknowledge the scanner deficiencies and either accept them or work around them. The size of some of the bariatric subjects challenged the scan field dimensions. For very large males possessing an extended abdomen, their physical dimensions would exceed the algorithm's capability to produce an accurate, measureable body model. Morbidly obese women can tend to have very large and ptotic breasts, challenging the ability of the scanner measurement software to extract accurate bust measurements. Fortunately, the scanner vendor worked with us to enlarge the scan field, and to allow certain measurement points to be manually adjusted. Still, the variability of the body shapes of morbidly obese individuals can tax the scanner's ability to generate a usable body image. Some measurements from a particular scan image may simply be unusable. The sheer size of the bariatric subjects warranted special consideration. The footprints glued onto the carpet of the floor mat within the scanner were designed for more normal sized individuals. They had to be removed and extended. Some of the measurements extracted from the body model, particularly biceps, elbows and forearms often were incorrectly located and needed to be manually adjusted. This was because many of the morbidly obese females had large arms that even when extended failed to separate completely from the torso.

If measurement errors occurred as part of the measurement process, and other measurements appeared accurate, the scan still was utilized. If the scan was unusable, a note was given to the patient to get rescanned if possible. The same would be true of postoperative scans.

The second item was to declare that all bariatric patients must be scanned. It was no longer an option. This might not seem to be an important point, but it is. Although the number of bariatric patients who voluntarily traveled to the plastic surgery center for their scans grew substantially since its trial phase, it was still a fraction of the total patients that underwent surgery. This was understandable due to the inconvenience. However, there would be instances of patients that already had their surgery coming to get scanned for the first time. This was an issue since there were no preoperative scan measurements available for comparison. In similar vein, when patients were told that body scanning was an option, some would simply consider it an additional burden to be avoided, sometimes to their regret.

The item addressed next was the training of the nurses. Good measurements start with knowing how to correctly capture a scan, how to identify a usable can, and what to do when the scan needs to be retaken. The key to introducing a new piece of equipment into the daily routines of a medical practice staff is to emphasize the simplicity of the device from the operator's standpoint. No one wants to be 
burdened with additional work. If it is too complex, cumbersome or unreliable, it will be avoided or operated incorrectly. Worse, it could affect personnel morale, as we briefly saw at the plastic surgery clinic.

The nurses were gathered together, and all the nurses were trained to take scans. One nurse received more comprehensive training. This individual would have responsibilities for training continuity, answer questions from other nurses and deal with the inevitable personnel turnover. Emphasis was placed on the simplicity of taking a scan, if only a few rules were followed. The nurses were told that this was a white light surface scanner, the equivalent of a group of people surrounding someone and turning flashlights on or off at different times. They were told that anything black or dark colored absorbs the projected light and that it wouldn't reflect back into the cameras to capture a picture. They were told about how the scanner searches for landmarks on the body to determine measurement location, and that it was because of this that the legs and arms need to be separated from the torso in order to find the crotch point and armpits.

But mostly, they were told not to be frightened or nervous when operating the machine. It wasn't mission critical and no one would be harmed by its usage. Moreover, all they had to do after the patient followed the rules and entered the scan chamber was to check or ask if they were positioned properly, the drapes were shut and then enter the patient's identifier in the name field. After that, it was simply a matter of selecting the "Start Autoscan" icon. The machine would do the rest of the work.

The bariatric clinic adopted a simple naming convention. A simple "Scan Information Sheet" was produced that the patient could fill out before they had their scan. The first iteration of this form requested their name, address, email address and height and weight. There was a check box to indicate whether the patient wanted the printout sent via email, or via the postal service. The nurse would use the name the patient filled in on the scan information form, and append to the name either "PREOP" or, in the case of a postoperative scan of the same patient, the length of time that had lapsed since their surgery. Examples would be "John Smith PREOP", or John Smith 3 MO POST, etc. Scans would be retrieved, matched by name, processed and distributed to the patient by our company.

Female bariatric patients were to be generally scanned in the nude, or had the option to be scanned in their bottom undergarment should it be light and solid colored in nature. Men were to be either scanned in the nude, or would be scanned in their jockey-type underwear if present. If wearing boxer undershorts, they were asked to remove them prior to getting scanned. It was important to tell the patients why this is necessary. The nurses were reminded to explain to the patient that this was a surface scanner, and that any clothing that draped over the body or hair that was hanging below the neck would distort the surface image and result in errant measurements.

The new NX-16 scanner at the bariatric practice was located within an examination room at the end of a hallway. It was positioned in such a way that when the door was opened, the subject looked straight at the scan chamber entrance. The computer display, located on a computer stand was just left of the chamber entrance was also directly visible. As scanning bariatric patients at the new facility with the NX-16 began, it was noticed that the "point cloud" image of the last patient scanned was often present on the display monitor when a new patient entered the room. The next release of scanner software incorporated a "screen clear" icon in the scanner software toolbar. As part of their scan routine, nurses allowed the patients to view their scan image after they dressed if they wished, but would use the "screen clear" icon once completed. In that way, any concerns about patients viewing other patient scans by accident was eliminated.

A coat rack and a chair the size and strength capable of seating a morbidly obese person was placed near the scanner. A small desk with a shallow open container was available for the patients to place their jewelry or other valuables. On the computer stand was a carton of disposable hair bands, and attached to one of the shelves of the computer stand were multiple hair clips. These were to be used by patients who needed to place their hair up to expose their neck. A full length mirror was installed on the back of the entrance door so that subjects could check their appearance after they put their clothes back on. 


\section{Lessons learned from installing a $3 \mathrm{~d}$ body scanner at the bariatric surgery practice}

Accept that some of the morbidly obese patients will have poor scan results. It is possible for a bariatric patient to be larger than the scan field dimensions. Some measurement extractions could result in a measurement error. If so, disregard that measurement and use the others. Some patients may be so large, or of such a shape that the scanner software may not create a measurable body model. If so, wait until the first postoperative visit and scan the patient again. Use that scan as the initial scan for documenting the physical measurements of the patient and note that it is a postoperative scan.

Make scanning patients mandatory. Do not make it optional. This will ensure that scanning patients will become part of the routine work flow of the clinic. Make sure the nurses that escort the patients to the various examination rooms know that it is their responsibility to take a scan of the patient at some point during their clinic visit.

Train the nurses or medical assistants who will operate the scanner as a group. Select one of them to get additional training and designate that person as the "train the trainer" point of contact who will be responsible for contacting the vendor and also for training subsequently hired personnel in the event of turnover.

Refresh their training on a quarterly basis. Have a small sign posted near the scanner computer reminding them of the simple rules required to capture a usable scan. Have the vendor point of contact posted near the scanner computer as well.

Keep the naming convention and sign-in sheet as simple as possible. Let the patient fill out the form, but check that the writing is legible! This is especially true for the e-mail address.

Clear the scanner computer display screen after the patient has put their clothes back on and exits the room. This will ensure that the next patient entering the room to get scanned will not see the scan image of the previous patient.

\section{A brief description of pitfalls that have been encountered}

Following the guidelines outlined above, from the start will, for the most part, assure that scanning the patient becomes a routine part of the staff's daily duties.

Inevitably, even though guidelines are written and the staff trained, unexpected items will pop up that need to be addressed. Once corrected, they then become part of the daily routine.

Here are some examples of "pitfalls" from past experiences:

Make sure that the cleaning crew does not bump into the scanner with the vacuum cleaner when vacuuming the scanner room. This has happened, to the point where the vacuum cleaner used by the cleaning crew knocked the scanner out of calibration.

Make sure that the bariatric patient doesn't lean against the scanner when getting dressed. Try to keep the coat rack and chair far enough away to prevent this. There have been times when a very large man or woman used the scanner wall for support while putting their clothes back on. The scanner will go out of calibration and that will cause all sorts of processing problems.

Make sure that the cables that connect the scanner cameras to the scanner computer are not too taught. This has occurred, too. A nurse needed to power on the scanner computer after an operating system update. The computer was under the computer stand and was hard to reach. The nurse pulled the computer forward to reach the power button and a cable that connected a ring of sensors in the scanner became unplugged. This prevented the scanner software program from initializing, resulting in a service call. 


\begin{abstract}
Make sure that the scan on the computer monitor is reviewed by the operator prior to releasing the patient from the scan chamber. On several occasions, a patient has been scanned with their feet facing in the wrong direction. Since the default display is in "point cloud" mode, the fact that the patient was facing backwards during the scan process is hard to notice.
\end{abstract}

If manually entering a date in the name field, make sure no special characters are used. There have been several frustrating occasions whereby the nurses, particularly recent hires, were entering the date in the name field of the scanner incorrectly. Instead of dd.mm.yy, one would use $\mathrm{dd} / \mathrm{mm} / \mathrm{yy}$. The use of the " $/$ " in the name field would result in the scan image being displayed but not saved to the hard drive since the operating system was looking for a storage path that did not exist. This was probably the most frustrating "pitfall" we ever encountered, since the patient could view their scan on the display but for all practical purposes their scan data was lost.

Insist on a manual scan log. Don't count on the scanning system software as the only log for documenting who was scanned. Have a simple columned sheet on a clipboard. The columns should at least have the patient's name, the date of the scan, the time of the scan, and the initials of the operator who took the scan.

The last item it turns out, is essential. We have experienced a situation where the scans appeared to be saved at random, with many scans not being saved at all. By comparing the manual scan log to the scans saved by the computer we found that a recent hire was simply told to enter the date after the patient name in the scan field. She entered the date with "/" special characters, thinking nothing of it. The scan would be processed and displayed, but wouldn't be saved out to the disk. This situation was corrected, and all the staff "re-briefed" as how to enter information into the scanner software name field.

\title{
"Current" 3D scanning...the KX-16 and SizeStream scanners at the bariatric practice today
}

In 2012, the bariatric practice moved from a section of a floor of a hospital to their own building. A room was dedicated to the scanner. The NX-16 system was removed and installed at the plastic surgery clinic. A KX-16 was installed at the new facility. The KX-16 utilized Primesense near-field infrared cameras instead of white light. This image quality appeared to be about the same as the older NX-16 scanner model. The big difference was one of convenience to both the nurses and the patients. It took far less time to acquire a scan on the KX-16. Instead of 1 minute, it took around 10 seconds. And since the sensors changed, it was no longer necessary to turn off the lights in the room or make sure that the privacy curtain did not let any light into the scan chamber.

In March of 2014, the KX-16 was removed and deployed at a research university. It was replaced by a SizeStream 3D scanner. There were minor changes to the guidelines developed for scanning bariatric patients at the clinic. They were mainly how to operate the new scanner.

The main changes to accommodate the SizeStream scanner were made back in our data center. The SizeStream scanner creates an .obj file instead of the TC2 .rbd format. A converter program back in the data center converts the .obj to a .wrl file. This .wrl file is then further subdivided to get enough point density so that the TC2 data converter program can create a sufficiently large .bin file.

We still use the TC2 desktop software as our main measuring software. So the .bin file is converted to an .rbd file and is measured and processed using the same systems that have processed the original scans, the NX-12 scans, the NX-16 scans and the KX-16 scans. 
Figure 3 displays the SideStream scanner installed at the bariatric clinic today.

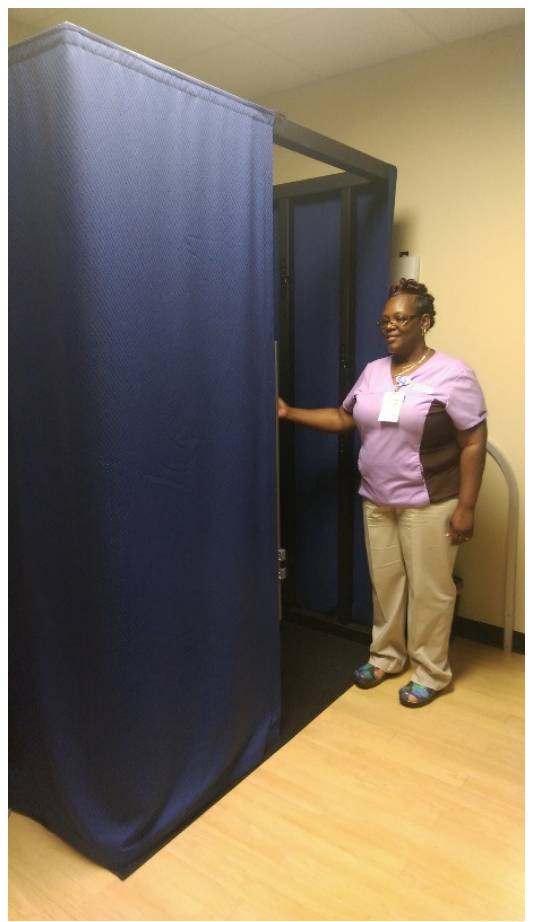

Figure 3. The SizeStream scanner at the Bariatric Clinic

Figure 4 shows that convenience furniture, mirror and privacy curtain that has been installed.

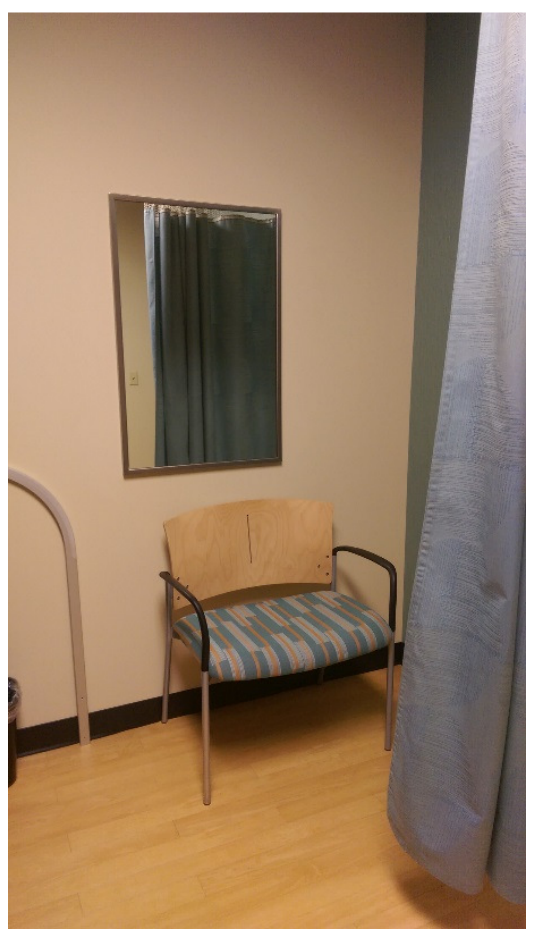

Figure 4. Simple, Private and Convenient Furnishings in the Scanner Room

The patient draws the privacy curtain that has been installed in the scanner room shut. There is a large chair, mirror and clothes tree (not shown). The patient keeps the privacy curtain drawn and then enters the scanner as depicted in Figure 5. 


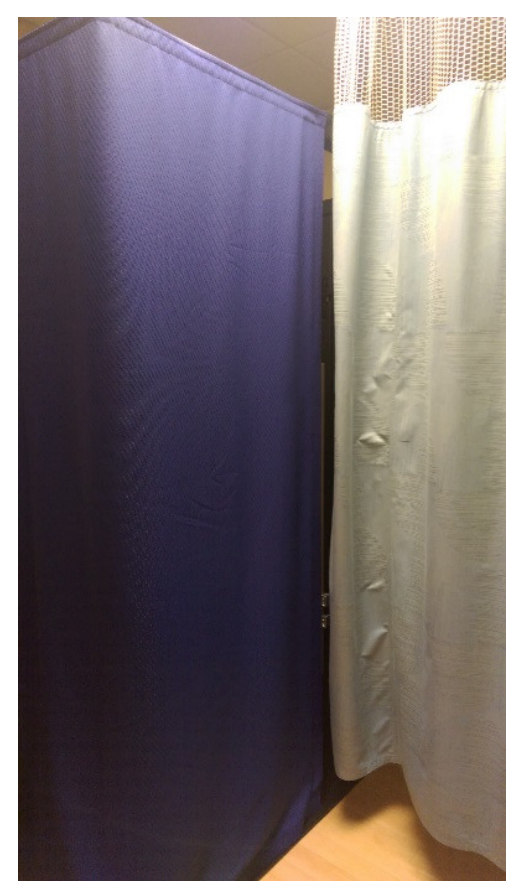

Figure 5. Patient directly enters SizeStream Scanner with Privacy Curtain Drawn Shut

Once the nurse checks that the patient is in the scan chamber and is standing in the correct position, she enters the name of the patient in the name field on the display and starts the scan process. Figure 6 depicts the nurse at the computer station while the patient is in the SizeStream scanner.

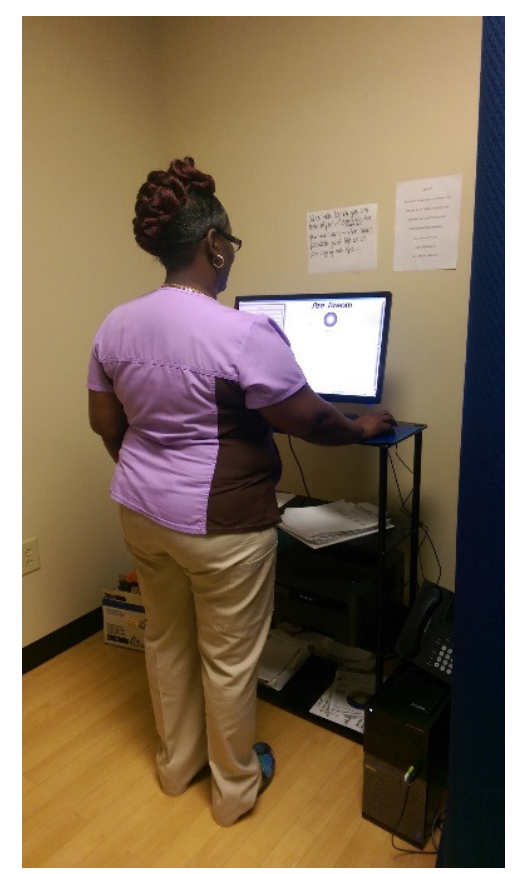

Figure 6. The Nurse (operator) at the SizeStream Computer Station

The time to acquire the scan is fairly quick, around 15 seconds. However, it takes some time for the SizeStream software to produce and measure the body mesh. The nurse generally tells the patient to relax while the scan is being processed. Once the body mesh is displayed, the nurse has a good idea as to whether the scan needs to be retaken or can be used as is. The nurse then tells the patient to get dressed and leaves the room. The entire scan process, from entering the room and the patient disrobing to acquiring the scan and having the patient get dressed generally takes around 10 minutes on average. 


\section{A word on billing}

At present, there are no insurance codes for this type of scanning. Contracts between medical entities and the company are individually negotiated. They are based on average expected yearly volumes of new patients. Invoices detailing the number of new patients scanned during a particular month, as well as returning patients are enumerated on the invoice. Payment for scan services is received in the form of a fixed rate sum on a monthly basis, regardless of the particular number of scans taken in the monthly period.

\section{Conclusions}

This paper has been an attempt to collapse 12 years of scanning experience in a medical environment into a few short, descriptive pages. It is the author's hope that the information presented herein, though sometimes a bit dry, will be helpful to those wishing to explore fielding a scanner within a medical practice.

The key is to make the scanning process a routine part of the duties of the clinic's staff. It is not necessary to have dedicated personnel to operate the scanner. All clinic staff who routinely deal with patients should be able to operate the scanner effectively, using a clear set of guidelines that everyone understands.

Scanning technology continues to progress at a rapid rate. The author has no doubt that other scanning devices, including hand held scanners will become commonplace within a clinical environment. Establishing and following clear guidelines and processes will ensure that new technology can be introduced without disrupting the workflow and the daily routines of a busy medical practice.

As a final thought, the author wishes to leave you with a photograph of the plastic surgeon who came up with the idea of utilizing a 3D scanner within a medical practice.

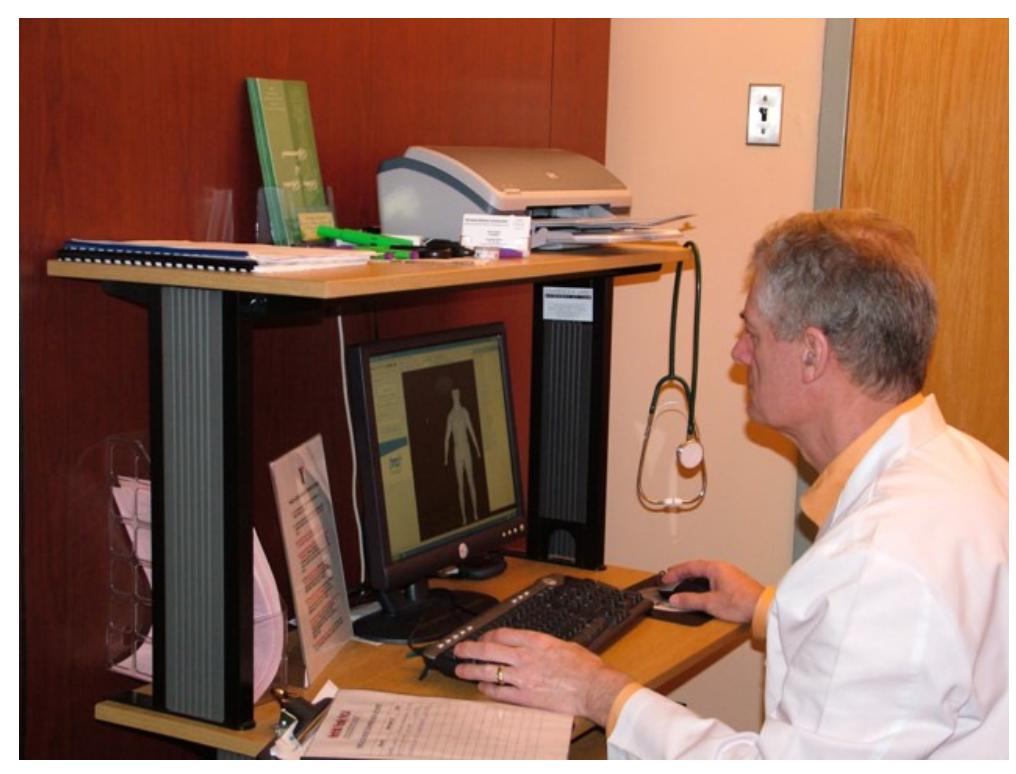

Still hard at work! 